Luther Street Medical Centre for the Homeless and Vulnerably Housed, Oxford, UK

2 Department of Primary Care, University of Oxford, Oxford, UK

3 St Michael's Hospital Department of Family and Community Medicine and Inner City Health Associates, Toronto, Ontario, Canada

Correspondence to: A Moscrop

Cite this as: BMJ 2020;371:m4150 http://dx.doi.org/10.1136/bmj.m4150

Published: 24 November 2020

\section{If social determinants of health are so important, shouldn't we ask patients about them?}

\section{Patients' socioeconomic circumstances should be routinely documented in their healthcare record, say Andrew Moscrop and colleagues}

\author{
Andrew Moscrop, ${ }^{1}$ Sue Ziebland, ${ }^{2}$ Gary Bloch, ${ }^{3}$ Janet Rodriguez Iraola ${ }^{3}$
}

Health inequities are worsening across Britain. Data from the Office for National Statistics (ONS) show that men in England's most deprived areas die almost a decade earlier than those living in affluent neighbourhoods. ${ }^{1}$ For women, life expectancy is falling in deprived areas. During the coronavirus pandemic, the strong emerging relation between covid-19 death rates and area deprivation reported by $\mathrm{ONS}^{2}$ and Public Health England ${ }^{3}$ has shown the exacerbation of existing inequities and highlighted the need for more comprehensive datasets in order to understand and reduce them.

The measurement and description of health and social inequities tends to rely on data at the level of geographical areas rather than individuals. We consider the limitations of using geographical data and look at how the collection of individual level socioeconomic data by healthcare systems could help to deliver care that is responsive to patients' social contexts and generate an enhanced dataset for research and monitoring of healthcare equity.

\section{The problem with postcodes}

Between the extremes of life expectancy exposed by the ONS data above, researchers in the UK have described a "social gradient in health." ${ }^{4}$ On this gradient, lower socioeconomic position or greater deprivation is associated with increased cardiovascular and cancer mortality $5^{6}$ and more mental health problems. ${ }^{7}$ Recognised social determinants of health such as employment status, occupation, education, housing, and household income not only affect people's health ${ }^{8}$ and health experiences ${ }^{9}$ but also their access to healthcare, outcomes of care, and engagement with health protection activities. ${ }^{10-13}$ Yet the UK's National Health Service has no system for routine recording of patients' socioeconomic circumstances. Social determinants are not routinely asked about, documented, or coded in clinical settings. The health service is, in effect, blind to those social determinants of health and healthcare.

When patient level socioeconomic data are required, composite deprivation scores are commonly used. These scores are based on averaged area data, inferred from the postcode of the patient's home address. Area based deprivation scores are then applied to individuals and used in clinical research, service evaluation (including the GP patient survey run by Ipsos MORI for NHS England), and even some clinical decision making (including cardiovascular risk calculation tools).
Although geographical data and area based deprivation scores may be appropriate for comparing groups, they cannot be used to characterise individuals from within those groups (ONS states that its Index of Multiple Deprivation should not be used for this purpose). ${ }^{14}$ Living in a "deprived area" is not the same as being deprived. For this reason and others (box 1), a patient's postcode is an unreliable indicator of their socioeconomic circumstances. The socioeconomic components of research, evaluation, and clinical decisions that use postcode data therefore have their reliability undermined too.

Box 1: Problems with using socioeconomic data derived from postcodes

- UK "postcode" data are drawn from larger geographical areas for which statistics are (LSOAs). An average postcode contains 15 households; an average LSOA contains $672 .{ }^{15}$ The larger averaging effects make the data much more liable to blunt extremes of affluence and deprivation, as well as blunting their measured effects

- Composite deprivation scores (including Indices of Multiple Deprivation, Townsend scores, Underprivileged Area Scores, and the Carstairs index) derived from postcodes conceal the social causes of health effects and obscure potential points of intervention

- Area data may identify an area of deprivation but do not show the deprivation of an individual from that area

- Confusion with area effects: postcodes may be associated with deprivation but may also be independently associated with other environmental health determinants

- Unreliability where populations are heterogeneous or undergoing rapid demographic change

- Inapplicability to mobile communities and the vulnerably housed in the UK, ${ }^{16}$ some of the most deprived and vulnerable members of society

\section{Duty to ensure equity}

Lack of robust individual level socioeconomic assessment in healthcare settings means that doctors are unable to identify people whose socioeconomic circumstances increase their risk of adverse health outcomes or treatment failures. Accordingly, it is not possible to identify those who may benefit from care plans acknowledging their social context, or to reliably monitor socioeconomic inequities in healthcare. available-usually lower layer super output areas estimated 320000 people who are homeless or 
We have an ethical duty to ensure that patients enjoy equal healthcare access, experience, and benefit. But UK systems that rely on patients' postcodes are simply too blunt to help us identify, address, and monitor socioeconomic inequities.

The World Health Organization has emphasised the importance of monitoring equitable service coverage across wealth and education gradients as part of achieving universal health coverage. ${ }^{17}$ Michael Marmot, one of the UK's leading public health researchers, has also advocated using markers of health equity such as socioeconomic position and education to monitor health and healthcare as a first step in confronting inequities. ${ }^{18}$ The British Medical Association suggests that doctors should consider patients in the context of social factors, noting the adverse health effects of poor housing, unemployment, poverty, and low educational achievement. ${ }^{19}$ But these recommendations have not been incorporated into UK policy, or led to any appreciable shift in practice, and the subject has been largely neglected in the UK research agenda.

The irony is that even when they are not acknowledged overtly, patients' socioeconomic circumstances and their health effects are unavoidable, especially in primary care. ${ }^{20}$ General practitioners spend large amounts of consultation time on non-medical issues relating to welfare benefits, housing, or unemployment. ${ }^{21}$ But the socioeconomic circumstances and difficulties discussed are rarely or only inconsistently recorded. Health practitioners' questions and their documented "social histories" continue to focus on socially influenced behaviours such as diet, exercise, alcohol consumption, and smoking habits.

By not asking patients about their social circumstances or recording socioeconomic data, doctors help to conceal these problems from public view and from the political agenda. These are important but often unacknowledged moral choices (box 2). Ending the complicity of the medical profession in health and healthcare inequities begins with data gathering: documenting the social contexts that affect patients' health and care outcomes, thereby evidencing inequities, aiding research into their causes, and monitoring changes.

\section{Box 2: Implicit moral choices in routine care: QRISK}

The routine management of cardiovascular disease (CVD) risk in UK primary care provides an instructive example of our problematic clinical response to patients' socioeconomic contexts. The National Institute for Health and Care Excellence (NICE) advises clinicians to "use the QRISK2 risk assessment tool to assess CVD risk for the primary prevention of CVD in people up to and including age 84 years." 22

Most of the UK population will therefore have periodic QRISK assessments in general practice. The QRISK algorithms use patients' postcodes to determine their neighbourhood deprivation score, which influences the calculated CVD risk and subsequent decisions about the need for blood pressure drugs and statins.

Leaving aside concerns about using postcodes to define individual deprivation, implicitly this is an attempt to ameliorate socially determined disease risk using clinical interventions, with an increased risk of side effects and harms for people who are more deprived. Despite a recent emphasis on shared decision making, the risk assessment typically occurs without a discussion about social health determinants between patient and doctor.

\section{Learning from US and Canada}

The lack of attention paid to social determinants in UK clinical care compares poorly with healthcare systems elsewhere that are beginning to take patients' socioeconomic contexts more seriously. In the US, during the Obama healthcare reforms, it was recognised that identifying and meeting patients' social needs could restrain healthcare spending and improve health outcomes. The US National
Academy of Medicine delivered specific recommendations for practitioners on the assessment and recording of patients' education level and their experience of financial hardship. ${ }^{23}$

Subsequent research has shown the feasibility of routine assessment of these and other social health determinants in US healthcare settings. ${ }^{24}$ Clinicians have reported useful adaptations in patients' care plans, ${ }^{25}$ improved communication, ${ }^{26}$ and that patients referred onward through social prescribing networks benefit from better access to community resources. ${ }^{27} 28$

Evidence is still awaited on the ultimate health and cost effects of socioeconomic assessments and social referrals in the US. Nonetheless, UK practitioners might take note. Social prescribing is already part of the NHS long term plan for England. ${ }^{29}$

Characterised as embracing everything from debt counselling for the insolvent to dance classes for the lonely, social prescribing is intended to link patients with suitable non-medical resources from the voluntary or community sector. The aim is to meet social needs while reducing pressures on primary care services. The NHS long term plan includes recruitment of hundreds of social prescribing link workers and anticipates the benefits to millions of patients. If this initiative is to meet the needs of the most deprived people and respond usefully to inequities and adverse social conditions, reliable individual level socioeconomic data are needed.

In Toronto, Canada, routine collection of patients' social data, including housing and household income, has been introduced in four large health organisations (including the family health team at St Michael's Hospital, where two of us are based). Doctors have used these data alongside an online tool to identify social benefits for which patients may be eligible, ${ }^{30}$ tackling income and related issues directly in the clinic (box 3). The socioeconomic data are also used to monitor healthcare access and outcomes, creating an improved basis for reducing healthcare inequities. ${ }^{31}$ Researchers have used the data to show that patients on low incomes are significantly less likely to participate in cancer screening (notably, this association was not found when only neighbourhood level data were used for the analysis). ${ }^{32}$ The relevance to the UK is clear, evidenced by the greater cancer mortality among patients from deprived areas attributed to "healthcare system factors," including later diagnosis and impaired access to optimal treatment. 33

\section{Box 3: Patient perspective-talking about income makes sense}


registration forms or by healthcare providers, with sensitivity and respect to ensure that patients feel comfortable and empowered.

Clear processes must be in place for data use and how to respond to patients identified as facing socioeconomic challenges. National coordination might be best, although socioeconomic questioning could prove valuable even if initiated locally. Information obtained could be used in auditing and resolving local inequities (for example, in relation to screening uptake, specialist referrals, or treatment follow-up).

Once identified, patients whose socioeconomic circumstances put them at risk of poor health and care outcomes can be flagged for enhanced care plans and targeted for specific interventions. For example, patients may be offered referral to local social prescribing networks, enhanced follow-up for chronic disease management, and additional information and encouragement to engage with health protection initiatives such as cancer screening and vaccines; they could also be given longer appointment times for discussion of health issues. In hospitals, patients with socioeconomic risk factors might receive enhanced discharge planning to reduce readmissions. Other healthcare teams that might make use of socioeconomic information for planning patient care and evaluating service equity include those in the Improving Access to Psychological Therapies programme.

Further research may be required to confirm the best approaches, such as the most appropriate questions to ask and how often to ask them. It will be important to seek the views of a range of patients. Adequate electronic record templates and clinical codes to record socioeconomic information will also be necessary. In primary care, Quality and Outcome Framework payments could be used to encourage the collection of socioeconomic data (as previously occurred for ethnicity data), with materials and messages developed to communicate the purpose to patients and staff, guidance produced on how best to audit and use the data, and the value of socioeconomic information emphasised in postgraduate training.

Doctors can make these changes happen. So why have they not done so already and why have socioeconomic inquiries been widely neglected? The omission may not be entirely unintentional. Our lack of process for asking about and documenting patients' socioeconomic circumstances may reflect learnt professional priorities, limited perceptions of the role of healthcare, and perhaps the relative socioeconomic privilege of doctors. ${ }^{35}$

Some cautions and caveats have been raised around asking about socioeconomic circumstances. ${ }^{36}$ These include concern about a lack of time, reflecting pressures on healthcare staff, and the prioritisation of biomedical factors over social determinants. Another common concern is the supposition that patients would object, yet so far there is little evidence of this in the US and Canada. Moreover, midwives in the UK already routinely ask every expectant mother about financial security, employment status, occupation, education, and housing. It is a collective failing that the children born to some of those mothers will have their lifespan diminished by as much as a decade because of socioeconomic circumstances. As life expectancy at birth for the most deprived people in the UK falls, as socioeconomic health inequities widen, and as inequities in healthcare continue to be overlooked, it is time for doctors to act on the knowledge that social determinants affect people's health.

\section{Key messages}

- Socioeconomic factors such as education, employment, occupation, housing, and household income affect patients' health as well as their healthcare access and outcomes
- These social determinants are not routinely documented in UK healthcare settings

- Robust individual socioeconomic assessments could facilitate supportive interventions for those whose social circumstances leave them at risk of poor health

- Social prescribing in primary care would be more equitable and effective when combined with routine documentation of patients' socioeconomic circumstances

- Monitoring healthcare access and outcomes using individual level socioeconomic data would create a basis for fairer healthcare

Contributors and sources: AM has global experience of working with marginalised groups, including refugees, people in prison, and those experiencing homelessness. SZ is programme director of research for patient benefit at the NIHR. Her areas of especial research interest include: how patient narratives can stimulate service improvements and better clinical training; and how the internet is changing healthcare and relationships with health professionals. GB is an associate professor in the University of Toronto's Department of Family and Community Medicine, focusing on the intersection between social risks to health and front line healthcare. JRI is a patient at St Michael's Hospital's family health team in Toronto and a lived experience expert patient adviser for their social determinants of health committee. For over 10 years she has been an advocate for people experiencing disabilities or poverty in Toronto. This article was drafted by AM following discussion with SZ, who provided comments, edits, and amendments. GB commented on an earlier draft, provided additional material, and reviewed and approved the final version. JRI reviewed and commented on an earlier draft and contributed the patient perspective

Competing interests: We have read and understood BMJ policy on declaration of interests and have no relevant interests to declare.

Provenance and peer review: Not commissioned; externally peer reviewed.

We thank Danny Dorling for useful discussion, Grainne Ferrari for confirming the current practices of midwifery teams, and Maurice Herson for reviewing and revising the final text. AM was supported by an NIHR in practice training fellowship 2015 to 2017. SZ is a NIHR senior investigator. GB is supported by a Phoenix fellowship from the AMS Foundation. The views expressed are those of the authors and not necessarily those of the NHS, the NIHR, or the Department of Health and Social Care.

Office for National Statistics. Health state life expectancies by national deprivation deciles, England: 2016 to 2018. 2020. https://www.ons.gov.uk/peoplepopulationandcommunity/healthandsocialcare/healthinequalities/bulletins/healthstatelifeexpectanciesbyindexofmultipledeprivationimd/2016to2018

2 Office for National Statistics. Deaths involving COVID-19 by local area and socioeconomic deprivation: deaths occurring between 1 March and 17 April 2020.

https://www.ons.gov.uk/peoplepopulationandcommunity/birthsdeathsandmarriages/deaths/bulletins/deathsinvolvingcovid19bylocalareasanddeprivation/latest

Public Health England. COVID-19: review of disparities in risks and outcomes. 2020. https://www.gov.uk/government/publications/covid-19-review-of-disparities-in-risks-and-outcomes Marmot MGAJ, Goldblatt P, Boyce T, McNeish D, Grady M, Geddes I. Fair society, healthy lives: strategic review of health inequalities in England post-2010. Marmot Review, 2010.

Mackenbach JP, Cavelaars AE, Kunst AE, Groenhof F. Socioeconomic inequalities in cardiovascular disease mortality; an international study. Eur Heart /2000;21:1141-51.

doi: 10.1053/euhj.1999.1990 pmid: 10924297

6 Cancer Research UK, National Cancer Intelligence Network. Cancer by deprivation in England: incidence, 1996-2010, Mortality, 1997-2011. NCIN, 2014.

Stansfeld SA, Head J, Fuhrer R, Wardle J, Cattell V. Social inequalities in depressive symptoms and physical functioning in the Whitehall II study: exploring a common cause explanation. J Epidemiol Community Health 2003;57:361-7. doi: 10.1136/jech.57.5.361 pmid: 12700221

8 Marmot MG, Smith GD, Stansfeld S, etal. Health inequalities among British civil servants: the Whitehall II study. Lancet 1991;337:1387-93. doi: 10.1016/0140-6736(91)93068-K pmid: 1674771

9 Hemingway H, Nicholson A, Stafford M, Roberts R, Marmot M. The impact of socioeconomic status on health functioning as assessed by the SF-36 questionnaire: the Whitehall II Study. Am J Public Health 1997;87:1484-90. doi: 10.2105/AJPH.87.9.1484 pmid: 9314801

10 Dixon A, Le Grand J, Henderson J, Murray R, Poteliakhoff E. Is the British National Health Service equitable? The evidence on socioeconomic differences in utilization. J Health Serv Res Policy 2007;12:104-9. doi: 10.1258/135581907780279549 pmid: 17407661

11 Laudicella M, Siciliani L, Cookson R. Waiting times and socioeconomic status: evidence from England. Soc Sci Med 2012;74:1331-41. doi: 10.1016/j.socscimed.2011.12.049 pmid: 22425289

12 Lindquist A, Kurinczuk JJ, Redshaw M, Knight M. Experiences, utilisation and outcomes of maternity care in England among women from different socio-economic groups: findings from the 2010 National Maternity Survey. BJOG2015;122:1610-7. doi: 10.1111/1471-0528.13059 pmid: 25227878

13 Cookson R, Propper C, Asaria M, Raine R. Socio-economic inequalities in health care in England. Fisc Stud 2016;37:371-403doi: 10.1111/j.1475-5890.2016.12109.

14 Department for Communities and Local Government. The English Index of Multiple Deprivation (IMD) 2015-guidance. 2015. https://www.gov.uk/government/statistics/english-indices-of-deprivation-2015 
15 Office for National Statistics. 2011 census: population and household estimates for small areas in England and Wales, March 2011. ONS, 2012.

16 Butler P. At least 320000 homeless people in Britain, says Shelter. Guardian 2018 Nov 22. https://www.theguardian.com/society/2018/nov/22/at-least-320000-homeless-people-in-britainsays-shelter

17 World Health Organization, International Bank for Reconstruction and Development, World Bank. Tracking universal health coverage: 2017 global monitoring report. 2017. https://www.worldbank.org/en/topic/universalhealthcoverage/publication/tracking-universal-health-coverage-2017global-monitoring-report

18 Marmot M. Universal health coverage and social determinants of health. Lancet2013;382:1227-8. doi: 10.1016/S0140-6736(13)61791-2 pmid: 24120189

19 British Medical Association. Social determinants of health-what doctors can do. BMA, 2011.

20 Popay J, Kowarzik U, Mallinson S, Mackian S, Barker J. Social problems, primary care and pathways to help and support: addressing health inequalities at the individual level. Part I: the GP perspective. J Epidemiol Community Health2007;61:966-71. doi: 10.1136/jech.2007.061937 pmid: 17933954

21 Citizens Advice. A very general practice: how much time do GPs spend on issues other than health?2015. https://www.citizensadvice.org.uk/Global/CitizensAdvice/Public\%20services\%20publications/CitizensAdvice_AVeryGeneralPractice_May2015.pdf

22 National Institute for Health and Care Excellence. Cardiovascular disease: risk assessment and reduction, including lipid modification. Clinical guideline 181. Recommendation 1.1.8. 2016. https://www.nice.org.uk/guidance/cg181/chapter/1-Recommendations

23 Adler NE, Stead WW. Patients in context-EHR capture of social and behavioral determinants of health. N Engl J Med 2015;372:698-701. doi: 10.1056/NEJMp1413945 pmid: 25693009

24 Gold R, Bunce A, Cowburn S, etal. Adoption of social determinants of health EHR tools by community health centers. Ann Fam Med 2018;16:399-407. doi: 10.1370/afm.2275. pmid: 30201636

25 Chhabra M, Sorrentino AE, Cusack M, Dichter ME, Montgomery AE, True G. Screening for housing instability: providers' reflections on addressing a social determinant of health. J Gen Intern Med 2019;34:1213-9. doi: 10.1007/s11606-019-04895-x. pmid: 30993632

26 Tong ST, Liaw WR, Kashiri PL, etal. Clinician experiences with screening for social needs in primary care. J Am Board Fam Med2018;31:351-63. doi: 10.3122/jabfm.2018.03.170419. pmid: 29743219

27 Garg A, Toy S, Tripodis Y, Silverstein M, Freeman E. Addressing social determinants of health at well child care visits: a cluster RCT. Pediatrics 2015;135:e296-304 doi: 10.1542/peds.2014-2888 pmid: 25560448

28 Gottlieb LM, Hessler D, Long D, etal. Effects of social needs screening and in-person service navigation on child health: a randomized clinical trial. JAMA Pediatr 2016;170:e162521. doi: 10.1001/jamapediatrics.2016.2521. pmid: 27599265

29 Drinkwater C, Wildman J, Moffatt S. Social prescribing. BMJ 2019;364:11285. doi: 10.1136/bmj.l1285 pmid: 30923039

30 Pinto AD, Bondy M, Rucchetto A, Ihnat J, Kaufman A. Screening for poverty and intervening in a primary care setting: an acceptability and feasibility study. Fam Pract 2019;36:634-8. doi: 10.1093/fampra/cmy129. pmid: 30649280

31 Wray R, Agic B, Bennett-AbuAyyash C, et al. We ask because we care: the tri-hospital and TPH health equity data collection research project report. 2013. http://www.stmichaelshospital.com/quality/equity-data-collection-report.pdf

32 Lofters AK, Schuler A, Slater M, etal. Using self-reported data on the social determinants of health in primary care to identify cancer screening disparities: opportunities and challenges. BMC Fam Pract 2017;18:31. doi: 10.1186/s12875-017-0599-z pmid: 28241787

33 Rachet B, Ellis L, Maringe C, etal. Socioeconomic inequalities in cancer survival in England after the NHS cancer plan. BrJ Cancer2010;103:446-53. doi: 10.1038/sj.bjc.6605752 pmid: 20588275

34 Brcic V, Eberdt C, Kaczorowski J. Development of a tool to identify poverty in a family practice setting: a pilot study[published correction appears in Int J Family Med. 2015;2015:418125]. Int J Family Med 2011;2011:812182. doi: 10.1155/2011/812182 pmid: 22312547

35 Waitzkin $\mathrm{H}$. The politics of medical encounters: how patients and doctors deal with social problems. Yale University Press, 1991.

36 Moscrop A, Ziebland S, Roberts N, Papanikitas A. A systematic review of reasons for and against asking patients about their socioeconomic contexts. Int J Equity Health 2019;18:112. doi: 10.1186/s12939-019-1014-2 pmid: 31337403 